Voix et Images

voixetimages

\title{
La critique est faillible
}

\section{André Brochu}

Volume 14, numéro 3 (42), printemps 1989

Gabrielle Roy

URI : https://id.erudit.org/iderudit/200805ar

DOI : https://doi.org/10.7202/200805ar

Aller au sommaire du numéro

\section{Éditeur(s)}

Université du Québec à Montréal

\section{ISSN}

0318-9201 (imprimé)

1705-933X (numérique)

Découvrir la revue

\section{Citer cet article}

Brochu, A. (1989). La critique est faillible. Voix et Images, 14(3), 513-521.

https://doi.org/10.7202/200805ar d'utilisation que vous pouvez consulter en ligne.

https://apropos.erudit.org/fr/usagers/politique-dutilisation/ 


\section{Poésie}

\section{La critique est faillible}

\section{par André Brochu, Université de Montréal}

Trop rares sont les tentatives, au Québec, de réflexion soutenue sur la poésie, et il faut mentionner l'initiative de Michel Muir qui, dans son dernier livre intitulé Poésie: la noblesse du réell, aborde le sujet, à la fois comme universitaire et comme poète. Hélas! on est vite consterné par une problématique désordonnée, infantile, qui trouve ses seuls ressorts dans la polémique et le bavardage narcissique, à grand renfort de points d'exclamation; qui ne contient d'aperçus valables que ceux glanés chez quelques bons auteurs (heureusement cités). Pour le reste, on est gratifié d'affirmations creuses telles que: Je sais d'expérience exaltante que la Poésie conduit l' homme vers les sommets de son être, où la grandiloquence ne le cède qu'à la fatuité. Et des bourdes, des bourdes! Je cite: Valéry écrivait, dans un ouvrage intitulé Morceaux choisis...

Michel Muir se dit aristocrate, il entend restaurer la pratique de l'écriture dans sa majesté originelle (présentation de la page couverture), ce qui bien sûr ne veut rien dire - la majesté est un rince-bouche, non un concept; et ce roisoleil a des injures de chauve-souris pour dénoncer les poètes qui ne partagent pas son idéalisme et son goût des clichés.

À ceux qui aiment rire, je recommande en particulier le récit de la révélation, sorte de pascalienne nuit du mémorial, qui commence par une interrogation passionnée du stylo à bille et se termine par l'intuition que Dieu $m^{\prime}$ 'avait donné des organes pour que je m'en serve. De quoi faire rougir les modernes champions du corps-texte!

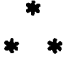

Tout en reconnaissant ses indéniables qualités, je n'avais pas beaucoup aimé Chambres, de Louise Dupré2. Mais Bonheur ${ }^{3}$, son dernier recueil, me séduit tout à fait. J'y vois même, dans l'ensemble, un maitre livre, où le bonheur est avant tout celui de l'expression, simple, limpide, capable de gravité et de profondeur, et d'infinies résonances: une merveille. Une parole qui touche, qui émeut, qui bouleverse même pour peu qu'on s'abandonne à son pouvoir de suggestion; car ces brèves proses et ces vers, toujours essentiels dans leur évocation des fragiles segments de l'existence quotidienne, nous mettent sur la piste de tout le vécu, déchirant de joie ou d'angoisse, d'amour décevant ou comblé. Surtout d'angoisse, surtout de tristesse: le titre est plutôt une antiphrase, non ironique cependant puisque le bonheur est possible et donne un sens à la vie, aux êtres: Le bonheur n' est pas l'envers de la mélancolie, mais son mur de lumière.

Mais peut-on goûter le bonheur autrement que comme l'autre de cette ombre, de cette catastrophe, de ce deuil qui sont le pain de chaque jour? 


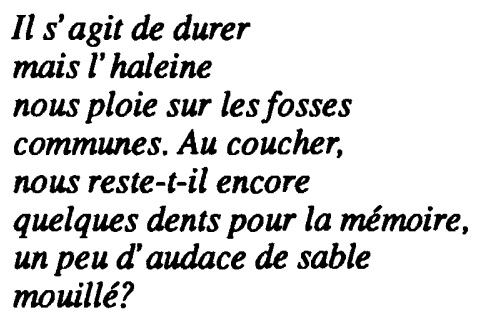

Le plus étonnant, c'est que, recouvrant le domaine entier de l'émotion, le langage soit exempt de pathos. Â aucun moment, il ne cesse de se donner pour du langage; de tenir à distance le référent, sans pour autant l'évacuer. Tout romantisme est évincé. Le frémissement sensible s'établit sur la base d'une parfaite pureté de forme - disons, pour employer un mot plus juste: de diction - qui laisse au lecteur toute liberté d'y consentir. On est à la fois loin des machines textuelles trop astucieuses, dont l'intérêt consiste surtout dans le fonctionnement du signifiant, et des platitudes que le récent souci du lisible a pu favoriser. On est loin aussi de cette prudence qui me semblait limiter la force d'expression du recueil précédent. Bonheur, c'est toute la femme livrée à son destin et qui l'affronte, mot à mot; qui dit, magnifique et douce et intraitable, à l'être aimé: Calme tu dors, tu dors, tu ne sais pas la gueule ouverte sur ma vie.

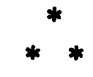

À vingt-deux ans, Guy Delahaye - pseudonyme de Guillaume Lahaise publiait les Phases, son seul recueil à l'exception d'un appendice canularesque qui s'intitule «Mignonne, allons voir si la Rose...» est sans épines (1912). Le tout est réédité aujourd'hui, avec plusieurs inédits, grâce à la piété filiale de Robert Lahaise, sous le titre d'Cuvres parues et inédites (la jaquette porte le titre d'CEuvres, tout simplement) 4 .

Moins substantielle, sans doute, que la poésie d'Eudore Évanturel dont j'ai parlé dans la précédente livraison, celle de Delahaye méritait elle aussi d'être portée à l'attention du public. Les Phases sont, il me semble, l'une des expressions les plus pures du symbolisme dans notre littérature. L'emploi d'une forme bizarre, les poèmes étant généralement constitués de trois tercets de vers de neuf pieds et regroupés en triptyques (que le poète s'entête à orthographier tryptiques), les thèmes évanescents, où s'affirment surtout le mépris du vulgaire et la condamnation du réel au profit de l'Idéal et du Rêve, imposent une personnalité littéraire aux antipodes du menu fretin nationaliste et terroiriste. Mais Nelligan, qu'admirait tant Delahaye, avait certainement plus à dire. Romantique, parnassien et symboliste tout ensemble, l'auteur du «Vaisseau d'on jouait de ces nombreuses voix et créait un monde complexe d'images et de rythmes. Delahaye, en un sens, est plus singulier, mais sa personnalité, écartelée entre le goût de la provocation et d'extraordinaires conformismes, notamment religieux, ne parvient guère à s'affirmer, et l'inspiration ne tarde pas à se tarir. Néanmoins on peut trouver du charme, et même une étrange beauté, à quelques poèmes comme celui-ci, intitulé «Doute»: 
Ce que le mal a d' aigu pour peines

Et le bien pour adoucissements,

Ce que tous deux ont de fortes chaines

Dans l' extase comme dans la haine,

Par un hymen de débordement

Où la monstruosité s'ajoute,

$S$ 'est accouplé dans une redoute,

$Q u$ 'atteignent, Néant, tes seuls amants,

Et de l' union jaillit le Doute

Expression alambiquée, certes, mais qui perturbe considérablement les routines et tend à faire du texte un pur signifiant, annonçant peut-être par là le formalisme moderne.

Mais gardons-nous d'ajouter à la liste des mouvements que Delahaye, si l'on en croit les critiques, aurait précédés, lui qui n'a pas su, par une activité soutenue d'écriture, rester son propre contemporain.

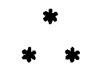

Quel ennui, d'être faillible! Sur mon bureau, là où j'enseigne, je garde en permanence un livre merveilleux: l'anthologie de Mailhot et Nepveu. Quand j'ai un moment libre, je l'ouvre au hasard et je lis. L'autre jour, je tombe sur un poème de facture classique mais qui, de façon tout à fait étonnante, me fait penser à Miron:

\section{Ô tous vos corps de lente usure}

[...] Vos mains de servitude et vos visages laids

[...] La teinte de vos chairs et le pli de vos traits

Et vos regards déshérités de l' aventure.

Mes compagnons, vous vivez tard dans ma mémoire.

[...] Et chaque soir et chaque nuit, je me sens las

De toute la fatigue éparse dans vos membres. [...]

C'était «Paroles aux compagnons», de Clément Marchand. Et je me suis souvenu du jugement rapide et plutôt sévère que j'avais porté sur les Soirs rouges, au moment de la publication d'un choix de textes où mes préférences allaient nettement au prosateur de Courriers des villages.

Être critique, c'est se tromper. Autant que possible... pas trop! Mais alors, dois-je une fois de plus braver l'opinion de raffinés collègues et affirmer mon goût pour la poésie de Claude Beausoleil? Quitte à m'attirer de fielleux sarcasmes, et au risque de me tromper une fois de plus, je dirai le bien que je pense de Grand Hôtel des étrangers ${ }^{5}$, qui n'est certes pas le livre le plus ambitieux de son auteur mais qui nous fait découvrir un autre aspect de son talent. Homme des grandes coulées lyriques, chantre néo-romantique d'une ville habitée et habitable, Beausoleil montre ici qu'il est aussi capable de concision et, à l'occasion de verve épigrammatique. 
Une sorte d'argument court en filigrane à travers les brefs poèmes du recueil - plus précisément, de sa section principale. Le poète a été invité à l'étranger, où il parle de lui-même et de ses amis; et il se heurte à la morgue de sinistres ganaches, qui l'entretiennent avec un infini mépris de la littérature qu'il est venu représenter.

Leurs voix râpant les pages

des livres de chez vous

sans accord et sans joie

ils buvaient par lapées

passéistes et sûrs d' eux

n'ayant pas trop de

temps a perdre

avec tous ces ouvrages

étranges venus d' ailleurs

Dans sa précision laconique, je dirais même son prosaïsme aigu, cette eauforte vaut bien un haiku. Car le haiku commence là où on attend du lyrisme qui ne vient pas. Mais on entrevoit ce qui apparaît mieux dans les autres poèmes: malgré les déconvenues, l'hostilité ambiante, tout ce qui fait de la vie une piètre aventure, Beausoleil garde l'espoir et le goût de poursuivre:

Ce que je veux comprendre

c'est la douleur du vide

le refus le mépris

et pourtant l'existence

Fanatique de ses amis, fanatique d'auteurs du passé trop vite relégués aux oubliettes - Clément Marchand, Robert Choquette, René Chopin -, fanatique de la ville et de ses coups d'aurore, aiguillonné pas sa si grande soif d'exister, Beausoleil, fantasque, éloquent, est un rare exemple de générosité: à la fois d'âme et d'écriture; et il est celui par qui le chant parfois existe, avec des accents d'une incomparable pureté:

Et si les pleurs sont souvent des écrans sans lueur

Il y a du temps à n'en pas douter pour tout cela

Et des accès nouveaux aux humeurs sans contrainte

S'illuminent et s'activent dans le plus clair du trajet

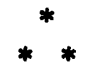

Les pierres, les poèmes. Ce sont les deux motifs principaux du beau recueil de Denuis Saint-Yves, Clandestin comme l'enfance ${ }^{6}$. Les pierres, de préférence celles de Forillon, ont quelque chose à voir avec la philosophie et avec la sainteté, avec le sable et avec le sang. Et les poèmes aussi. Je soupçonne les unes et les autres d'être interchangeables. En tout cas, les pierres sont des rétroviseurs où les feux de la nuit / brûlent des questions éternelles; et les poèmes ont des cheveux qu'il ne faut pas couper en quatre, car les émeraudes s'y trouvent. Les 
énigmes de l'être sont très simples, mais elles font du quotidien une continuelle surprise. Il n'y a rien là d'hermétique, car les poèmes ont autre chose à faire que / d'évacuer le sens ou non. Si les images de Denuis Saint-Yves ne livrent pas facilement leur secret, c'est qu'elles sont toumées vers l'intérieur, comme les pierres et comme les questions inépuisables de l'existence. La ponctuation, souvent irrégulière, s'emploie à casser le mouvement naturel de la phrase, à ouvrir des trappes sous les pas et mettre en évidence les mots:

\section{[...] les saisons sont là qui se comparent \\ aux saisons et, font front - commun - dans le grand déluge du temps qui dure [...]}

Couramment, le et est suivi, contre toute orthodoxie, d'une virgule qui dément la coordination. Dans l'univers de Saint-Yves, rien ne va de soi, et non pas à cause d'une anarchie des choses, d'une déréliction, mais parce que tout existe selon un ordre qui est celui de la plus grande profondeur. Matière et esprit ensemble: les pierres sont la philosophie / épurée de ses contextes maussades. j'y vois / des laines qui se souviennent. aussi, un / nid et, ses passagers. Très belle image, d'une origine (le nid) qui n'est pas fixation mais principe de mouvement, de passage.

Jocelyne Felx a une capacité de renouvellement remarquable. Les Pavages du désert ${ }^{7}$ sont aussi différents de Nickel-Odéon que ce dernier l'était d'Orpailleuse $^{8}$. Et l'épouvantable compacité thématique de Nickel-Odéon fait place ici à un discours beaucoup plus aéré, plus accueillant pour le lecteur même s'il reste hautement culturel, truffé de références à Léonard de Vinci et à Pascal, et à toutes sortes de savoirs pratiques et théoriques, qui vont de la philosophie à la géologie ou à la théorie musicale.

Il y a une aimable contradiction entre le goût de Jocelyne Felx pour tant d'aspects précis du réel et l'affirmation, maintes fois répétée, qu'il n'y a pas un grain d'espérance, que morte est Ithaque / et dépourvue d'espérance / cette Odyssée / terrestre. Sur le papier délicatement violacé, l'expression sobre et décidée du nihilisme perd de sa sécheresse, entre en consonance avec l'assertion contraire des puissances de vie:

il y a des écritures qui crient

des paroles qui sont des hommes

et des hommes qui sont des noms

il y a Pascal volant le frisson [...]

Optimisme et pessimisme sont les pôles et, plus encore, les moteurs du texte, qui déploie entre eux des imaginations brillantes dont les motifs sont empruntés à tout un magasin de conceptions ingénieuses, celles des grands inventeurs et des esprits universels. De discrets plagiats (du genre des plagiats dits «nécessaires») démultiplient le sens: 


\section{[...] et le bourgeonnement des axes}

distraitement dociles

à quelque fin profonde monte [...]

Vinci, constamment présent, se prolonge en Valéry, le temps d'une allusion, et d'un raisonnement: car le poème, sur sa láncée optimiste, raisonne beaucoup, et n'acquiert son juste contrepoids d'émotion que dans le désenchantement postérieur, seule ouverture pratiquée du côté de la commune condition mortelle.

La seule, vraiment? Non: il y a aussi la tendresse passionnée, celle qu'inspire, dans la première section du recueil, une jeune musicienne, Rose, dont le nom irrigue tout le discours du monde. Dans la seconde section, Mona Lisa (oui, la Joconde de Vinci) reprend un peu le même rôle symbolique mais avec la distance inhérente à la référence culturelle, ce qui me semble plus conforme au génie de l'auteur. L'émotion ne peut éclore tout à son aise que sur le terreau de ce qu'il faut bien appeler une pensée - chose plutôt rare en poésie.

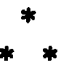

D'Anne-Marie Alonzo, deux livres: une «fiction», Seul le désir', et un recueil, le Livre des ruptures ${ }^{10}$, qui porte pour la première fois le sous-titre "poésie»; texte important, qui rappelle par son ampleur et sa beauté Bleus de mine et Ecoute, sultane.

Au fait, les fictions étaient déjà poésie, et le Livre des ruptures rappelle beaucoup les recueils antérieurs. Peut-être la syntaxe est-elle moins bousculée. La poésie serait affaire de continuité. La phrase, tout en conservant un caractère profondément inventif, se fait beaucoup plus chantante, envoûtante, par exemple, dans l'extraordinaire récit lyrique intitulé «Brune orchidée noire», dont les alinéas sont comme de longs versets commençant tous par le pronom «elle». La magie moyen-orientale de l'apologue s'y joint à une acuité de sentiments tout occidentale.

C'est d'amour, d'un amour bien ancré dans le désir, que nous entretiennent inlassablement les textes d'Anne-Marie Alonzo, avec une ferveur chaque fois renouvelée, qu'il s'agisse de l'élan par lequel le moi s'enlace à l'autre avec d'infinies précautions ou de sauvages ardeurs, ou des inévitables ruptures, vectrices de mort:

Il n'y a que toi! et la douleur je mourrai d l'instant

j' implorerai serai facile à traîner, je dirai: donne! sans sup-

plier t'écrire ne servirait qu'à bannir et bannie je m'éloigne

te laisse toute encore à désirer.

Ce qui frappe, dans ces lignes comme dans l'ensemble des deux recueils, c'est l'omniprésence du vocabulaire de la réalité humaine, quotidienne, des relations interpersonnelles, des activités les plus simples. Très peu de références à la nature - représentations ou métaphores -, très peu d'objets. La matière est essentiellement corps, corps de l'autre et corps propre, et l'univers se réduit 
à la distance qu'il y a du moi à l'être aimé. De là l'importance du travail sur la syntaxe, qui vient ouvrir la relation humaine au mystère en la complexifiant à l'infini, par des déplacements, des glissements qui lui donnent l'opacité même de cette chose, de cette nature refusées sur le plan thématique:

Douloureusement tendre tu me regardes! de ton ceil s'effrite l'or en voyant que? de moi vois-tu qui tant

t'effraie après sept ces années je t'aime n'ai pas changé $[. .$.

Le discours, qui pourrait être banal, de l'éternel plaidoyer amoureux acquiert ainsi l'ampleur propre à la tragédie ou au cantique, quand l'amour devient complètement et désespérément humain.

une fois de plus

on s'enfouit dans sa vie

comme un exercice

en attendant

Et une fois de plus, Bernard Pozier publie un recueil, Ces traces que l'on croit éphémères ${ }^{11}$, où se côtoient le bon et le moins bon - non le meilleur ni le pire car, dans ses moments les plus inspirés, là où le poème devient charme, grâce fragile, il ne saurait susciter l'enthousiasme. La métaphysique qui le porte a quelque chose de trop attendu, d'infiniment quotidien:

est-ce que je sais certains soirs

ce que j'écris et pourquoi

Cela peut être touchant, sympathique, mais il est rare que l'écriture nous entraîne dans quelque labyrinthe où prenne chair soudain la grâce d'une image. «Le tain du paysage» y parvient, qui évoque ces fenêtres de la fiction où l'on se reflète, où le réel même nous reflète dans la fragilité de nos failles / quand le verre se défait / et que la vitre ne déforme plus le monde / ni l'être qui regarde à travers elle / vers lui-mêmé. Réussite trop peu fréquente. À côté, on se heurte à cette platitude d'un couple où ton corps est presque aussi moi que le mien qui est lui-même presque aussi toi que le tien; androgynie, pensez donc, qui $n$ 'est qu' un des autres noms de l' amour.

On se heurte surtout à des paronomases produites mécaniquement, sans nécessité: Malgré mes mains / mes manauvres et mes manettes (!); quand les paumes nous pâment, etc. Quand cessera-t-on de confondre le travail sur le signifiant avec ces amusements primaires?

Mais malgré ces côtés décevants, qui nous font comprendre que, dans le registre de la lisibilité, Pozier a peu de choses à dire, le recueil marque un net progrès sur les Bacilles de tendresse, d'éprouvante mémoire ${ }^{12}$.

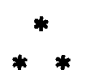


De la poésie de Gilbert Langevin, on pourrait dire qu'elle ne change guère et que, pourtant, elle renouvelle chaque fois son message d'espoir et de crainte, de révolte et de tendresse (thèmes emblématiques, mentionnés en page couverture du dernier recueil). Au plaisir ${ }^{13}$, paru il y a plus d'un an, donne à lire d'étonnantes échappées vers la lumière:

c'est dans un saut fervent

que le sang couve ses apogées

parmi des constats beaucoup moins enthousiastes:

aimer tourne de l'aile

avant d' aller périr

au large du malfroid

sombrent ainsi

les plus tendres éclats

La joie l'emporte cependant, ce qui fait de ce recueil l'un des plus sereins de Langevin. La Saison hantée ${ }^{14}$, plus récent, reprend l'alternance du clair et du sombre, mais avec prédominance de ce dernier. Le poème, qui nous donne habituellement la quintessence concrète du monde en quelques vers brefs et suggestifs, accueille parfois l'allusion politique, soit qu'il stigmatise les menteurs, gens du pouvoir qui font régner la loi du peu, ou qu'il nomme ces hauts lieux de la terreur que sont Port-au-Prince ou d'autres scènes de la charcuterie planétaire. L'engagement, parce qu'il est limité à quelques poèmes, est d'une grande efficacité et se propage, par connotation, à l'ensemble des textes. D'autre part, la fraîcheur et la simplicité des images tient souvent du prodige, comme dans ces vers:

le repos te va

comme un vent sucré

ou

le calme laisse couler

un joli cours d' air

vers qui réinventent la prosodie sur la base d'une vérité et d'une fluidité incomparables. Quelques mauvais jeux de mots, à côté de cela (faible mais fiable / sur fil de fable), du reste astucieux, ne dérangent rien: l'inspiration existe.

Pour conclure, j'attire l'attention des amateurs de poésie sur les publications suivantes: Forêt vierge folle, de Roland Giguère ${ }^{15}$, réédité en «Typo» - une fête d'images graphiques et verbales; le numéro spécial des Herbes rouges intitulé 20 ans ${ }^{16}$, qui commémore un impressionnant anniversaire avec des témoignages, souvent intéressants, de ceux qui, au fil des ans, ont alimenté la revue; enfin, un essai critique de Pierre Nepveu, l'Écologie du réel ${ }^{17}$, qui réexamine la tradition littéraire, et en particulier poétique, du Québec, surtout depuis 
Saint-Denys Gameau et Alain Grandbois, et qui étudie la dialectique de la poésie et de la prose à travers quelques auvres importantes de nos modernités successives. Un ouvrage clé pour la compréhension de l'écriture présente et des mouvements qui l'ont précédée.

1 Michel Muir, Poésie: la noblesse du Réel, essai, Montréal, Louise Courteau, 1988, $200 \mathrm{p}$.

2 Louise Dupré, Chambres, Montréal, les Éditions du remue-ménage, 1986, 92 p. Cf. mon compte rendu dans Voix \& images, vol. XIII, no 1, automne 1987, p. 167-168.

3 Louise Dupré, Bonheur, Montréal, les Editions du remue-ménage, 1988, 104 p.

4 Guy Delahaye, Euvres, édition critique par Robert Lahaise, Montréal, Hurtubise HMH, 1988, $406 \mathrm{p}$.

5 Claude Beausoleil, Grand Hotel des étrangers, Trois-Rivières, Écrits des Forges/ Europe Poésie, 1988, 60 p.

6 Denuis Saint-Yves, Clandestin comme l'enfance, Trois-Rivières, Ecrits des Forges, $1988,74 \mathrm{p}$.

7 Jocelyne Felx, les Pavages du désert, Saint-Lambert, le Noroît/la Table rase, 1988, $94 \mathrm{p}$.

8 Jocelyne Felx, Orpallleuse, Saint-Lambert, le Noroit, 1982, 70 p.; et Nickel-Odéon, Saint-Lambert, le Noroît, 1985, 174 p. Cf. mes comptes rendus dans Volx \& images, vol. VIII, no 3, printemps 1983, p. 504 et vol. XII, no 1, automne 1986, p. 139.

9 Anne-Marie Alonzo, Seul le désir, Montréal, NBJ, 1987, 60 p.

10 Anne-Marie Alonzo, le Livre des ruptures, Montréal, l'Hexagone, 1988, 124 p.

11 Bemard Pozier, Ces traces que l'on croit éphémères, Trois-Rivières, Ecrits des Forges/la Table rase, 1988, 74 p.

12 Bemard Pozier, Bacilles de tendresse, Trois-Rivières, Ecrits des Forges, 1985, 110 p. Cf. mon compte rendu dans Voix \& images, vol. XI, no 3, printemps 1986, p. 556-557.

13 Gilbert Langevin, Au plaisir, Trois-Rivières, Ecrits des Forges, 1987, 60 p.

14 Gilbert Langevin, la Saison hantée, Trois-Rivières, Ecrits des Forges, 1988, 80 p.

15 Roland Giguère, Forêt vierge folle, Montréal, l'Hexagone, 1988, 218 p. (Typo).

16 En collaboration, 20 ans, les Herbes rouges, non numéroté, Montréal, 1988, 84 p. On y trouve, entre autres, des textes de Roger Des Roches, Lucien Francceur, André Roy, François Charron, Normand de Bellefeuille, André Gervais, Paul Chamberland, Marcel Labine, France Théret, Hugues Corriveau, Carole Massé, André Beaudet, Jean-Marc Desgent, Rosie Harvey, Louise Bouchard et Jean-Yves Soucy.

17 Pierre Nepveu, l'Écologle du réel - Mort et naissance de la littérature québécoise contemporaine, Montréal, Boréal, 1988, 243 p. 